\title{
Photodynamic Disinfection in the Treatment of Chronic Adult Periodontitis: A Multicenter Clinical Trial
}

\author{
Roger C Andersen* and Nicolas G Loebel \\ Regulatory \& Clinical Affairs at Ondine Biomedical, Medical laboratory in Vancouver, Canada
}

Submission: March 16, 2017; Published: September 06, 2017

*Corresponding author: Roger C Andersen, Regulatory \& Clinical Affairs at Ondine Biomedical, Medical laboratory in Vancouver, Canada, Tel: 425489-110; Fax: 425-489-1101; Email: randersen@ondinebio.com

\begin{abstract}
Background: Scaling and root planing (SRP) has been used effectively in periodontal therapy for more than 100 years, but produces only modest, transient reductions in bacterial load. Photodisinfection is a bactericidal modality based on the generation of oxidative stress on outer bacterial cell membranes, when light of a matched wavelength illuminates a photosensitive agent near the membrane. The aim of this study was to investigate the effect of photodisinfection as an adjunct to SRP in chronic adult periodontitis.
\end{abstract}

Methods: 121 patients with chronic periodontitis were recruited into a multicenter, randomized, examiner-blinded, parallel-group study. All patients were treated with scaling and root planning (SRP) at all pocket probing sites $\geq 5 \mathrm{~mm}$ which exhibited bleeding on probing (BOP). Following SRP the patients were randomized to a no further treatment arm or an antimicrobial photodynamic therapy (aPDT) arm. The primary endpoint of this study was clinical attachment level (CAL). Secondary endpoints were probing pocket depth (PD) and BOP. aPDT was carried out using a diode laser of $670 \mathrm{~nm}$ and $0.2 \mathrm{~W}$ in power. A methylene blue based photosensitizer was used for bacterial staining.

Results: Compared to the control arm, the aPDT arm carried a significant improvement in clinical attachment level. aPDT with methylene blue caused a significant decrease in pocket probing depth. These results were apparent at 6-weeks as well as the 12-week follow-up period.

Conclusions: A PDT using a methylene based photosensitizer shows a distinct improvement in clinical attachment levels and pocket depth over scaling and root planning alone in the treatment of chronic periodontitis.

Keywords: Periodontitis; Apdt; Bacterial infections; Photodynamic therapy; Photodisinfection; Scaling \& Root planning

\section{Introduction}

Scaling and root planing (SRP) has been used effectively in periodontal therapy for more than 100 years $[1,2]$, but produces only modest, transient reductions in bacterial load [3,4]. Up to two-thirds of dental patients carry microbes incorporating antibiotic resistance genes, the expression of which can impair systemic or locally-administered adjunctive antibiotic therapy [5]. Photodisinfection is a bactericidal modality based on the generation of oxidative stress on outer membranes of prokaryotes, when light of a matched wavelength illuminates a photosensitive dye near the membrane. This approach avoids the generation of resistance in the target microorganism [6], primarily because the rapidly bactericidal action is generated via physical cell wall destruction rather than interference with biochemical pathways. The aim of this study was to investigate the effect of photodisinfection as an adjunct to SRP in the treatment of chronic adult periodontitis.

Antimicrobial Photodynamic Therapy ("aPDT") has been demonstrated to be an effective, non-antibiotic, antimicrobial approach in vitro [6-9]. The technique involves the use of light energy to activate a photosensitive molecule which then either transmits energy directly to a substrate via electron abstraction/ redox interactions (type I photoreaction) or interacts with molecular oxygen to produce singlet oxygen and derived compounds (type II photoreaction) [10]. Certain photosensitizers are also effective histological stains, and this property confers a degree of binding specificity to the molecule, localizing the bactericidal effect to prokaryotes. The photoreactions generated upon absorbance of light by such dyes have been shown to kill microbial cells primarily via lipid peroxidation and membrane damage mechanisms [79]. The nanosecond lifetime of singlet oxygen in biological media limits the diffusion of the molecule and thereby further localizes the bactericidal effect, as does the limited penetration and scattering of the activation light in human tissues.

aPDT has specifically been demonstrated to exert potent antimicrobial effect against biofilms, in contrast to many 
antimicrobial compounds that retain efficacy only against planktonic cultures $[10,11]$. Sessile bacterial biofilms are formed when colonizing microbes encapsulate themselves against a surface in an exopolymeric matrix composed of secreted polysaccharides, proteins, and nucleic acids. Proximity of one cell to another permits information exchange through quorum sensing [12] and the upregulated exchange of plasmid DNA, with the resulting gene transfer conferring survival advantages across the entire colony [13]. Studies have shown that biofilms of oral bacteria are much more difficult to eradicate by conventional means (i.e. antibiotics or debridement) than planktonic-form equivalents because of the strong tissue adherence and physical exclusion of antimicrobial substances in the biofilm form $[12,13]$. The fact that aPDT was found to be so effective against periodontal biofilms suggests an innate advantage over other antimicrobial periodontal therapies, and this has led to the proposal that aPDT may represent a promising alternative for the treatment of periodontal disease $[14,15]$. This work reports on the first multicenter, randomized, blinded, controlled evaluation of photodynamic disinfection as an adjunct to $\mathrm{SRP}$ in the treatment of chronic adult periodontitis.

\section{Materials and Methods}

This 121 patient, randomized, examiner-blinded, multicenter, parallel-group study was designed to assess the efficacy and safety of a aPDT in the treatment of adult patients diagnosed with chronic periodontitis, by evaluating gain in clinical attachment level (CAL), reduction in probing depth (PD), and reduction in bleeding on probing (BOP).

The study included two cohorts:

i. A control group, receiving SRP alone; and

ii. A test group, receiving SRP followed by aPDT treatment to the qualifying periodontal defect sites.

The aPDT system deployed in this study consisted of three basic components:

i. Alaser base station incorporating a low power $(<225 \mathrm{~mW})$, continuous-wave diode laser (Periowave ${ }^{\mathrm{TM}}$, Ondine Biomedical, Vancouver, BC) operating at a red wavelength $(670 \mathrm{~nm})$ over a 60-second pre-programmed treatment cycle.

ii. An autoclaveable handpiece connected to the laser via fiberoptic cable; and

iii. A treatment kit composed of a single-use light diffusing tip, a blunt-ended irrigation needle and a pre-filled syringe containing photosensitizer solution $(0.01 \%$ methylene blue USP in a buffered, isotonic, viscosity-modified base).

Methylene blue (3,7-bis[Dimethylamino]-phenazathionium chloride) is a cationic heterocyclic aromatic dye commonly used for histological visualization, treatment of methemoglobinemia [16], tracing lymphatic drainage during sentinel lymph node dissection [17], and visualization of GI dysplasia [18]. aPDT using methylene blue is widely used in European countries for the disinfection of fresh frozen donor plasma and red cell suspensions [19].
The aPDT treatment protocol consisted of scaling and root planning (SRP) with or without ultrasonic instrumentation, and subsequent irrigation of the treatment site with approximately $0.2 \mathrm{ml}$ of photosensitizer solution. The irrigated pocket was then illuminated via the laser handpiece for a period of 60 seconds. SRP was conducted per the standard protocols in use by each examiner, generally consisting of ultrasonic instrumentation after regional block anesthesia. Reverse-phase intrasulcular anesthetic agents and hemostatic agents were not permitted to be used in this study because of the unknown potential for interference with the photodynamic disinfection procedure. Double-pass clinical attachment level (CAL) measurements were made from the base of the sulcus to the cementoenamel junction (CEJ) using a PCPUNC-15 periodontal probe. Approximately 20-gram force (50 $\mathrm{N} / \mathrm{cm} 2$ ) probing force was used. Examiners were calibrated prior to study initiation. Occasionally the CEJ position with respect to the base of the sulcus was obscured when tissue overlapped the CEJ or a prosthetic crown was present. In the case of a crown, CAL measurements were taken from the crown margin to the base of the pocket. Where tissue overlapped the CEJ, the degree of overlap was assessed by probing between the free gingival margin and the CEJ, and the CAL measurement then made via calculation (pocket probing depth minus the extent of overlap). Gain in CAL was prospectively declared as the primary endpoint of the study.

Double-pass pocket depth (PD) measurements were taken by calibrated examiners using the same PCPUNC-15 periodontal probe. Bleeding on probing (BOP) was given a positive (present) or negative (absent) score. Positive BOP score required bleeding to occur within 15-30 seconds after probing, and be readily apparent. Reduction in PD and BOP were secondary endpoints of the study. All measurements were recorded at the mesiobuccal (MB), buccal/ facial (B/F), distobuccal (DB), mesiolingual (ML), lingual/palatal (L/P) and distolingual (DL) positions (6 sites per tooth). The study was reviewed and approved by the institutional review board (IRB) at each participating site, prior to enrollment of study patients. A written informed consent was signed voluntarily by each patient after the nature of the study was explained and prior to any study-related procedure being performed. The informed consent document was reviewed and approved by the Investigator, Sponsor, and the Investigator's IRB prior to initiation of the study. Two types of activation laser were deployed during the course of this study: a 150-mW unit (cohort $\mathrm{N}=15$ ), and a 220-mW unit (cohort $\mathrm{N}=106$ ).

\section{Enrollment Criteria and Patient Demographics}

Study sites were in Canada at the University of Alberta; the University of Western Ontario; the University of Saskatchewan; and a private practice in North York, Ontario. Patients diagnosed with chronic adult periodontitis with otherwise unremarkable medical histories, with at least 18 or more fully erupted teeth and with at least 4 measurement sites exhibiting pocket depth of 6-9 $\mathrm{mm}$ in at least two quadrants of the mouth were eligible to participate in the study. Subgingival instrumentation over the past 4 months disqualified the patient from enrollment, as did antibiotic use in the preceding 1 month period. Other exclusion criteria included known allergy to 
methylene blue, glucose-6-phosphate dehydrogenase deficiency by patient report, active periapical or periodontal abscess, history of acute necrotizing ulcerative gingivitis, pregnancy, or concomitant use of any photosensitizing medications.

Blinding was accomplished by utilizing a treatment-blinded dental examiner qualified to take clinical measurements who was separate from the treating clinician. The examiner collected all clinical measurement data at baseline and at the follow-up study visits. Each patient and clinician at each center was instructed to maintain confidentiality of the treatment status to the treatmentblinded examiner. Each study site was trained on the study protocol, data collection, and CRF completion procedures at an intensive study initiation visit prior to the enrollment of study patients. An independent contract research organization performed data entry. 121 patients in total were enrolled and randomized 1:1 to either "SRP+aPDT" or "SRP alone" arms. Patients enrolled in the "SRP alone" arm were required to go through a screening period of up to 16 days, two SRP treatment visits (one for each half of the mouth sequentially) and follow-up examinations at 6- and 12 weeks. Patients enrolled in the "SRP+aPDT" arm were required to go through a screening period of up to 16 days, one SRP treatment visit (for first half of the mouth), one SRP plus aPDT treatment visit (SRP for the second half of the mouth and aPDT for all selected treatment sites) and follow-up examinations at 6 and 12 weeks. The first 15 patients recruited into the study were treated with the $150-\mathrm{mW}$ laser unit (if randomized to the SRP+aPDT arm), or to the control arm for that cohort. The next 106 patients recruited into the study were treated with the $220-\mathrm{mW}$ laser unit (if randomized to the SRP+aPDT arm), or to the control arm for that cohort. Patients were evaluated at the screening visit, the two treatment visits (one half of the mouth scaled in each visit, aPDT treatment in the second visit, if applicable), and then at 6 week and 12-week follow-up visits.

Demographics for the patients are given in Table 1 below.

Table 1: Patient demographics.

\begin{tabular}{|c|c|c|c|}
\hline \multicolumn{2}{|c|}{} & $\begin{array}{c}\text { Control Group } \\
\text { (SRP alone) }\end{array}$ & $\begin{array}{c}\text { Test Group } \\
\text { (aPDT plus SRP) }\end{array}$ \\
\hline \multirow{2}{*}{$\begin{array}{c}\text { Number of } \\
\text { Patients }\end{array}$} & \begin{tabular}{c} 
Screening Visit \\
\cline { 2 - 4 }
\end{tabular} & $63^{*}$ & $58 \dagger$ \\
\cline { 2 - 4 } & $\begin{array}{c}\text { 6 Week Follow- } \\
\text { Up Week } \\
\text { Follow-Up }\end{array}$ & 55 & 55 \\
\hline \multicolumn{2}{|c|}{ Females (at screening) } \\
\cline { 2 - 4 } Age (Mean \pm SD)
\end{tabular}

*8 patients missed the 6 -week and 12-week follow-up visits.

†3 patients missed the 6-week and 1 missed the 12-week follow-up visits.

\section{Statistical Methodology}

As defined in the protocol, the primary analysis population (by ICH-E9 definition) included all randomized patients from whom a baseline value and at least one post-baseline measurement were generated. The primary analysis of safety included all patients who received randomized treatment and contributed postrandomization follow-up data. Safety endpoints were based on treatment received. This study incorporated one primary endpoint, clinical attachment level, along with the following assumptions: detection threshold for improvement $=0.4 \mathrm{~mm}$; power=90\%; significance $=95 \%$; SD of measurements $=0.63$. Type I error probability was set at 5\%, and type II error probability at $10 \%$. A two-sided study would have required 106 patients to reach $90 \%$ statistical power; with an assumed drop-out rate of $15 \%$, it was decided to recruit 121 patients.

The methodological recommendations of a recent publication [20] were adopted for the statistical analysis, because of the robust treatment of baseline interactions, statistical power, and randomization. Two different but similar methods, based on generalized linear models, were utilized for this analysis using commercially available software (SAS): Generalized Estimating Equations (GEE) and Mixed Models. Both methods correct for the dependency of observations between patients, i.e., both methods consider the fact that for each patient, data were taken as repeated measures in time (screening visit, week 6 and week 12) and space (2 measurements -PD and CAL -at 6 different points for up to 32 teeth). Mixed Models were used when the dependent variables were continuous variables (CAL changes, PD changes) and GEE were utilized when the dependent variable was binary (BOP).

In these mixed-model analyses, patient assessments were treated as repeated measurements, and baseline value for each site of measurement was regarded as a covariate. Initial tested fixed effects and interaction with the treatment effect included center effect (i.e. principal investigator effect), gender effect (male or female), smoking status effect (current, former, never, unknown) and alcohol consumption effect (current, former, never, unknown). Based on initial analyses of each individual trial, a significant interaction between center and treatment effect was expected, related to outcome differences between the various studies. Center was thus treated as a random effect. Alcohol consumption was also treated as a random effect, since the correlation between alcohol consumption and treatment effect was not readily interpretable and it has been well-established that alcohol consumption, as declared by the patient, is a poorly reliable parameter.

\section{Results}

Results of this study showed that patients receiving adjunctive aPDT utilizing the 220-mW laser unit experienced significantly better outcomes than SRP alone, when assessed by average CAL gain $(0.19 \mathrm{~mm}, \mathrm{p}<0.0001)$ or $\mathrm{PD}$ reduction $(0.15 \mathrm{~mm}, \mathrm{p}<0.0001)$ on defect sites with an initial probing depth $\geq 5 \mathrm{~mm}$ exhibiting BOP. For $P D$ reduction $>1 \mathrm{~mm}$, frequency distribution analysis demonstrated that adjunctive aPDT produced roughly twice the improvement in PD than SRP alone, even when large reductions ( $>4 \mathrm{~mm}$ ) were considered. When data from both the $150 \mathrm{~mW}(\mathrm{~N}=15)$ and 220 $\mathrm{mW}(\mathrm{N}=106)$ laser units were pooled however, results were compromised (CAL gain NS, PD reduction $0.08 \mathrm{~mm}, \mathrm{p}=0.0001$ ). This outcome demonstrated the importance of sufficient laser energy 
to activate the photosensitizer solution. No device-related serious adverse events were reported during or after the course of the study.

\section{Clinical attachment level gain}

CAL gain for those pockets with an initial probing depth $\geq 5 \mathrm{~mm}$ and exhibiting BOP was significantly better in the SRP+aPDT group than in the SRP alone group, when the 220 -mW laser unit was utilized (Table 2).

Table 2: CAL gain.

\begin{tabular}{|c|c|c|}
\hline & aPDT+SRP & SRP Alone \\
\hline $\begin{array}{c}\text { Number of pockets } \\
\text { (week 12) }\end{array}$ & 2349 & 2463 \\
\hline $\begin{array}{c}\text { Week 6 (LS } \\
\text { Means* } \pm \text { SE) }\end{array}$ & $-0.69 \pm 0.05$ & $-0.50 \pm 0.05$ \\
\hline $\begin{array}{c}\text { Week 12 (LS } \\
\text { Means } \pm \text { SE) }\end{array}$ & $-0.71 \pm 0.05$ & $-0.54 \pm 0.05$ \\
\hline $\begin{array}{c}\text { Global Treatment } \\
\text { Effect (p-value) }\end{array}$ & \multicolumn{2}{|c|}{$-0.19(\mathrm{p}<0.0001)$} \\
\hline
\end{tabular}

*8 patients missed the 6-week and 12-week follow-up visits.

†3 patients missed the 6-week and 1 missed the 12-week follow-up visits.

\section{Probing depth reduction}

PD reduction for those pockets with an initial probing depth $\geq 5 \mathrm{~mm}$ and exhibiting BOP was significantly better in the SRP+aPDT group than in the SRP alone group, when the 220-mW laser unit was utilized (Table 3).

Table 3: PD reduction.

\begin{tabular}{|c|c|c|}
\hline & aPDT + SRP & SRP Alone \\
\hline $\begin{array}{c}\text { Number of pockets } \\
\text { (week 12) }\end{array}$ & 2352 & 2460 \\
\hline $\begin{array}{c}\text { Week 6 (LS } \\
\text { Means* } \pm \text { SE) }\end{array}$ & $-0.82 \pm 0.04$ & $-0.69 \pm 0.03$ \\
\hline $\begin{array}{c}\text { Week 12 (LS } \\
\text { Means } \pm \text { SE) }\end{array}$ & $-0.85 \pm 0.04$ & $-0.68 \pm 0.03$ \\
\hline $\begin{array}{c}\text { Global Treatment } \\
\text { Effect (p-value) }\end{array}$ & $-0.15(\mathrm{p}<0.0001)$ \\
\hline
\end{tabular}

*LS Means corresponds to Means adjusted by considering the differences in number of teeth, pockets, etc, per patient.

\section{Bleeding on probing reduction}

Analysis of BOP reduction in per-protocol patients demonstrated no statistically significant differences between treatment arms at week 6 or at week 12 (Table 4).

Table 4: BOP percentage.

\begin{tabular}{|c|c|c|}
\hline & aPDT+SRP & SRP Alone \\
\hline $\begin{array}{c}\text { Pockets with BOP at } \\
\text { baseline }\end{array}$ & 1706 & 1552 \\
\hline Week 6 & $48 \%$ & $46 \%$ \\
\hline Week 12 & $50 \%$ & $50 \%$ \\
\hline $\begin{array}{c}\text { Global Treatment } \\
\text { Effect (p-value) }\end{array}$ & NS & \\
\hline
\end{tabular}

\section{Whole-mouth effect}

The positive effect of SRP on pockets not specifically treated (i.e. whole-mouth effect) has been well established in the literature; the effect is probably related to suppression of pathogen-translocation from an infected site to less-infected sites in the mouth. In this study, analysis of CAL gain and PD reduction for all pockets in the mouth (i.e., both treated and untreated pockets) showed a highly statistically significant improvement $(\mathrm{p}<0.0001, \mathrm{p}<0.0001)$ over that generated by SRP alone. This effect was found to be independent of baseline, measurement, tooth, tooth location, probing site, gender, alcohol use or smoking status.

\section{Adverse events}

No device-related serious adverse events were reported during or after the course of the study. There were no reports of tooth sensitivity.

\section{Frequency Distribution Analysis}

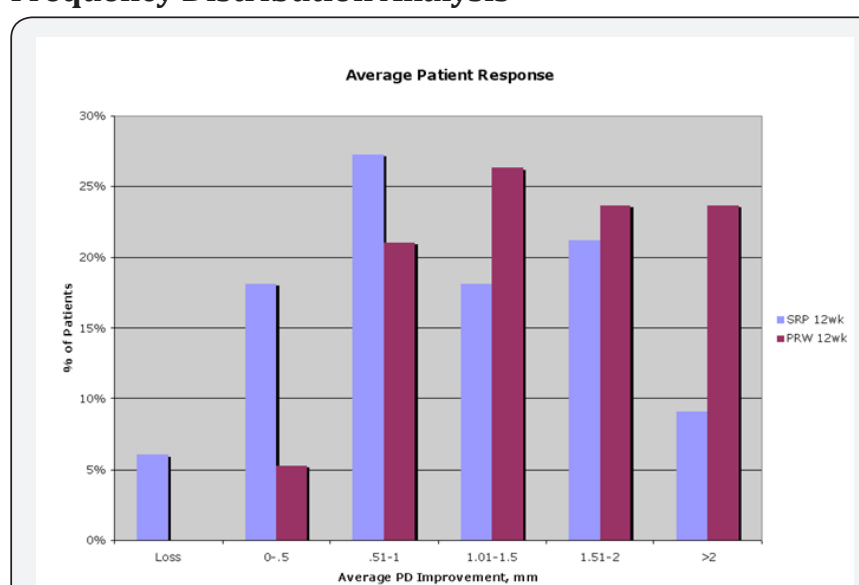

Figure 1: Average PD improvement, 12 wks, stratified in 0.5$\mathrm{mm}$ increments

The probing measurements are presented as frequency distributions, rather than means, to further analyze the results presented above. Figure 1 below demonstrates average patient response stratified by pocket depth improvement for adjunctive aPDT treatment vs. SRP Alone: Note that the pocket depth reduction associated with SRP peaked at approximately $1 \mathrm{~mm}$ at 12 weeks. The response due to adjunctive aPDT therapy is right-shifted towards larger pocket depth recoveries at 12 weeks, with approximately double the gain compared to SRP-alone at the $>2 \mathrm{~mm}$ recovery level. No patient treated with aPDT exhibited continued deterioration (loss) in pocket depth, whereas $>5 \%$ of the patients experienced such continued loss in the SRP-alone arm.

Figure 2 below presents similar stratification data, except that the data is expressed in terms of percentage of individual pockets responding rather than average response per patient: Adjunctive aPDT therapy produced enhanced responses at all pocket depth recovery levels in excess of the $1 \mathrm{~mm}$ reduction attributable to SRP alone. Substantially fewer pockets in the aPDT arm experienced continued deterioration (loss) compared to those in the SRP-alone arm. The odds ratio for improvement in PD recovery in the aPDT 
versus SRP-Alone cohorts is given in Figure 3. Note that up to $1 \mathrm{~mm}$ of average pocket depth recovery, the gains due to SRP are expected; above $1 \mathrm{~mm}$, adjunctive aPDT therapy produces increased benefit and on average, produces roughly twice the benefit of SRP even when large gains $>4 \mathrm{~mm}$ are considered. Note that up to $\sim 1 \mathrm{~mm}$ of average pocket depth recovery, the gains due to SRP are expected; above $1 \mathrm{~mm}$, adjunctive aPDT therapy produces increased benefit and on average, produces roughly twice the benefit of SRP even when large gains $>4 \mathrm{~mm}$ are considered.
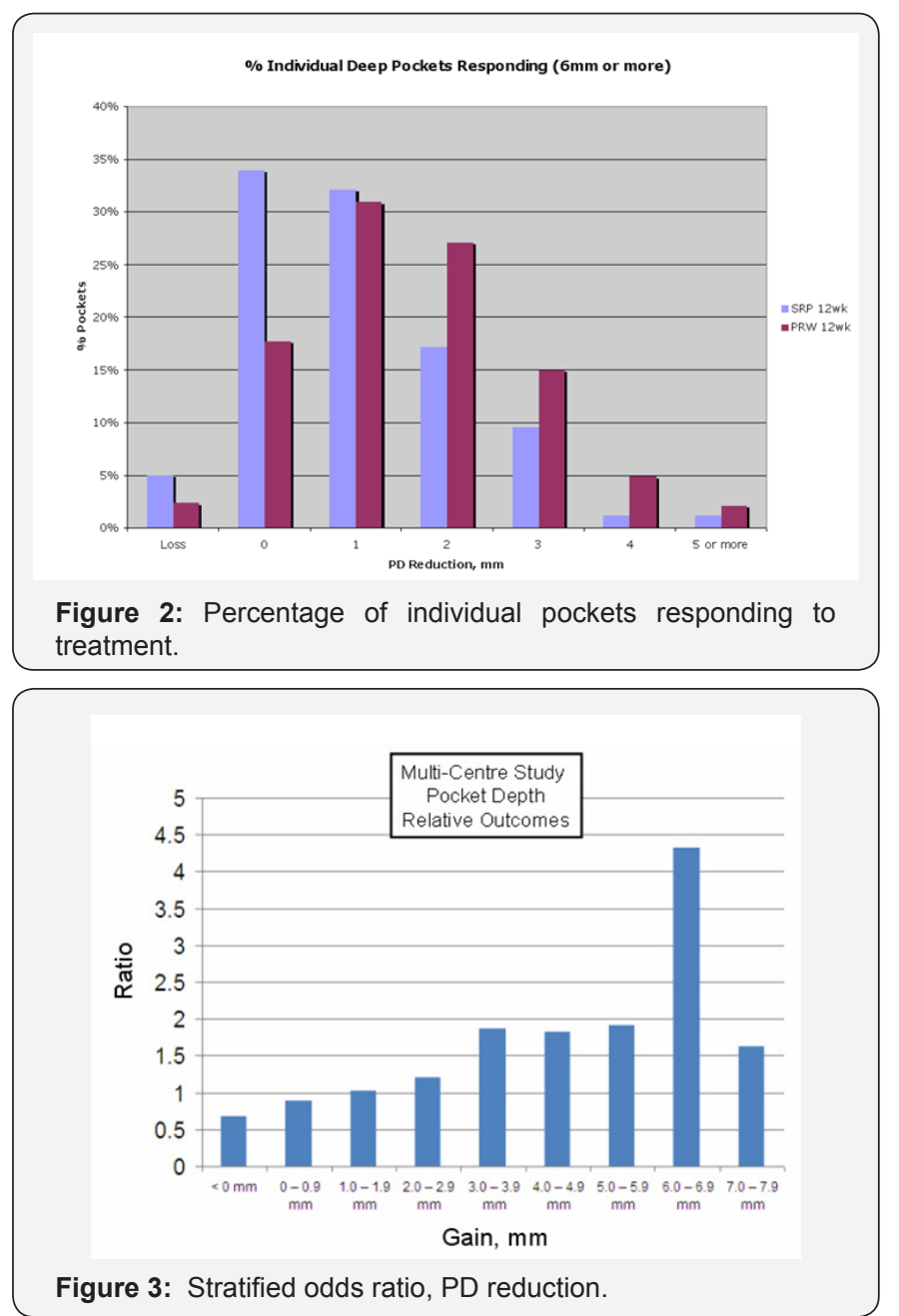

\section{Discussion}

Nonsurgical treatment of chronic adult periodontitis relies upon scaling and root planing as the gold standard of subgingival debridement [20]. While many patients respond well to comprehensive mechanical debridement, especially when accompanied by improvement in at-home oral hygiene procedures, there nevertheless exists a refractory population that continue to demonstrate chronic periodontal tissue breakdown. These patients often present with concomitant predisposing risk factors such as smoking, diabetes, hereditary factors, systemic disease and so forth; local factors such malocclusion, inadequate prosthetic preparation, or chronically persistent superinfection with one or more pathogenic species may also be involved [21]. Scaling and root planing has been demonstrated to leave both calculus [22] and bacteria [23] behind in the treated area, and to open dentinal tubules, permitting invasion by the residual periopathogens with subgingival recolonization shortly thereafter $[24,25]$. In the phenotypically susceptible host, a continued inflammatory response occurs that is paralleled by a sustained increase in gingival crevicular fluid (GCF) flow rate [26] as the local microvasculature reacts to proteolytic enzymes, cytokines, and other pro-inflammatory factors.

Locally-administered chemotherapeutic agents administered with a view to controlling etiologic microbes may be partially or wholly neutralized by GCF and constituents (e.g. chlorhexidine binding to anionic acid groups on glycoproteins [27] or by physical displacement through the rapid GCF exchange [28]). In addition, bacteria within the bulk layers of the plaque biofilm are metabolizing rather slowly, thereby muting drug uptake and response, and superficial layers of the biofilm may degenerate under the bacteriostatic influence of the antibiotic, inhibiting further diffusion into the bulk layers [29]. Maintaining appropriate mean inhibitory concentration (MIC) of any subgingivally-administered antimicrobial is difficult under these conditions, and requires either a slow-release matrix (days to weeks of exposure) or conversely, a rapidly cidal approach which thereby overcomes the problem of physical displacement from the sulcus. The aPDT approach falls into the latter category, demonstrating kill levels of 4-6 logs $(10,000$ $1,000,000$-fold) reduction in microorganism count in laboratory biofilms over 60 seconds [30]. In addition, aPDT treatment has been demonstrated to inactivate the virulence-associated protease of P. gingivalis, and to inactivate destructive host cytokines such as tumor necrosis factor-alpha (TNF- $\alpha$ ) and interleukin (IL)$1 \beta$ [31], providing a potent dual pathway to suppression of the microorganisms as well as the various pro-inflammatory factors involved. Another advantage of aPDT as a microbicidal modality is that it is not dependent on the issues of resistance that plague the use of antibiotics6. Microbial resistance to antibiotics is upregulated by the systematic, worldwide, improper use of antibiotics for treatment of non-susceptible human infections (e.g. viral infections), by inadequate administration regimens, and through use in animal feedstocks for growth promotion [32]. Gene patterns found in resistant bacterial strains are identical in animals and humans, indicating transfer of those resistance genes between the species . Low-dose (subantimicrobial) administration of antibiotics such as doxycycline for reduction of matrix metalloproteinase has been proven to upregulate antibiotic resistance [33]. The incidence of multiple drug resistance in many organisms is steadily increasing, in many cases with lethal effect (the first US case of vancomycinresistant staphylococcal infection was reported July 3, 2002 in Michigan [34]). While locally-administered sulcular antibiotics reduce the rate of systemic exposure, efficacy can be limited due to the sequestration and displacement factors discussed above. Adjunctive use of systemic or local antimicrobials can also result in allergic reactions, stomach upset, local discomfort and adverse staining or hypersensitivity reactions. Finally, another advantage of aPDT lies in the fact that the low-level laser light source does not cause thermal damage to tissues; by contrast, use of high-power 
lasers to physically debride tissues such as those of the gingival sulcus may be problematic, due to the side-effects of thermal injury and damage to surrounding tissues [35]. A retrospective analysis of available literature demonstrated no additional benefit of high power lasers used adjunctively to SRP for sulcular debridement purposes and this outlook is currently mirrored by the American Dental Association [36,37].

aPDT appears to be well-suited to the requirement for a potent antimicrobial adjunct to scaling and root planing, especially in the patient presenting with a concomitant risk profile for continued disease. Results of this multicenter clinical study demonstrated a wide therapeutic window. No patient compliance is required, but the technique does involve administration of the activation light for 60 seconds per defect site. Because a single tooth may present with more than 1 defect site (e.g. mesial to the buccal as well as to the palatal, or midline defects to either side of a furcation etc.), the total adjunctive procedural time throughout the mouth can represent an appreciable fraction of the time spent scaling and root planing. The present study required patients to undergo scaling and root planing by quadrant in each of four consecutive visits, to limit each appointment to a reasonable time. It should also be noted that excessive bleeding after the SRP procedure was correlated with reduction of additional benefit by aPDT, an effect probably related to physical ejection of the photosensitizer from the bleeding sulcus. Although generally not difficult to ensure, adequate bleeding suppression is required.

One secondary endpoint-reduction in BOP-did notreach significance, irrespective of laser power or protocol. This outcome matches that of other studies involving locally-administered antimicrobials. BOP has been demonstrated to provide poor positive predictive value for ongoing periodontal disease, and conversely, strong negative predictive [38]. From a clinical perspective, this implies that BOP does not imply ongoing periodontal disease, but rather presence of prior injury to the sulcular epithelium with inadequate or incomplete re-epithelialization at the time of repeated challenge with the probe. The gingival tissue, while apically reattaching to the dentinal surfaces of the planed root, remains parakeratinized and friable to a degree for quite some time after treatment and can therefore bleed when abraded. The clinical results above demonstrate that adjunctive Periowave significantly enhances gain in clinical attachment level, and reduction in pocket depth over SRP alone, but does not necessarily generate a more robust or competent re-epithelialization compared to SRP alone.

\section{Conclusion}

This is the largest study ever done on the use of aPDT in the treatment of chronic adult periodontitis. Results of the study have demonstrated the safety and efficacy of aPDT therapy in the nonsurgical treatment of chronic adult periodontitis. The laser power delivered to the treatment site was shown to be an important determinant of outcome, with only small differences (150 mW [9]) versus $220 \mathrm{~mW}$ [13.2 J]) producing significantly different outcomes in CAL gain. Attention to minimizing bleeding after SRP was also required. When utilizing the higher-power activating laser source, both gain in CAL and reduction in PD were demonstrated to be highly statistically significant over SRP alone, and this significance extended to both directly-treated pockets and to untreated pockets that presumably received indirect benefit from the disinfection protocol. Evaluation of the data using response frequency analysis demonstrated an improvement in these key clinical indices of approximately a factor of 2 over SRP alone.

\section{Disclosures}

This study was financially supported by Ondine Biomedical Inc., Vancouver, BC, Canada. Drs. Andersen and Loebel are employees of Ondine Biomedical Inc.

\section{References}

1. Aurelian L, Ono F, Burnett J (2003) Herpes simplex virus (HSV)associated erythema multiforme (HAEM): a viral disease with an autoimmune component. Dermatol Online J 9(1): 1.

2. Khalil I, Lepage V, Douay C, Morin L, Wallach D, et al. (1991) HLA DQB1*0301 allele is involved in the susceptibility to erythema multiforme. J Invest Dermatol 97(4): 697-700.

3. http://emedicine.medscape.com/article/1122915-overview.

4. Francesco D, Aurora P, Alfredo R (1995) Persistent erythema multiforme: Report of two new cases and review of literature. J Am Acad Dermatol 33(2): 366-369.

5. Maharani LA, Retno PR (2009) Immunopathological aspects of oral erythema multiforme. Dent J (Maj Ked Gigi) 42(4): 159-163.

6. Huff JC, Weston WL, Tonnesen MG (1983) Erythema multiforme: A critical review of characteristics, diagnostic criteria, and causes. J Am Acad Dermatol 8(6): 763-775.

7. Samim F, Auluck A, Zed C, Williams PM (2013) Erythema multiforme: A review of epidemiology, pathogenesis, clinical features, and treatment. Dent Clin North Am 57(4): 583-596.

8. Tennesson MG, Soter NA (1979) Erythema multiforme. J Am Acad Dermatol 1: 357-364.

9. Yetiv JZ, Bianchine JR, Owen JA (1980) Etiologic factors of the Stevens Johnson syndrome. South Med J 73(5): 599-602.

10. Margaret Lemak A, Madeleine Duvic, Samuel Bean F (1986) Oral acyclovir for the prevention of herpes-associated erythema multiforme. J Am Acad Dermatol 15(1): 50-54.

11. Weston WL, Brice SL, Jester JD, Lane AT, Stockert S, et al. (1992) Herpes simplex virus in childhood erythema multiforme. Pediatrics 89(1): 3234.

12.Lamoreux MR, Sternbach MR, Hsu WT (2006) Erythema multiforme. Am Fam Physician 74(11): 1883-1888.

13. Schalock PC, Dinulos JG, Pace N, Schwarzenberger K, Wenger JK, et al. (2006) Erythema multiforme due to Mycoplasma pneumoniae infection in two children. Pediatr Dermatol 23(6): 546-555.

14. Isik SR, Karakaya G, Erkin G, Kalyoncu AF (2007) Multidrug-Induced Erythema Multiforme. J Investig Allergol Clin Immunol 17(3): 196-198.

15. Mockenhaupt M (2011) The current understanding of Stevens-Johnson syndrome and toxic epidermal necrolysis. Expert Review of Clinical Immunology 7(6): 803-813.

16.Chang YS, Huang FC, Tseng SH, Hsu CK, Ho CL, et al. (2007) Erythema multiforme, Stevens-Johnson syndrome, and toxic epidermal necrolysis: acute ocular manifestations, causes, and management. Cornea 26(2): 123-129. 
17. Mockenhaupt M, Viboud C, Dunant A, Naldi L, Halevy S, et al. (2008) Stevens-Johnson syndrome and toxic epidermal necrolysis: Assessment of medication risks with emphasis on recently marketed drugs. The EuroSCAR-study. J Invest Dermatol 128(1): 35-44.

18. Ayangco L, Rogers RS 3rd (2003) Oral manifestations of erythema multiforme. Dermatol Clin 21(1): 195-205.

19. Brice SL, Krzemien D, Weston WL, Huff JC (1989) Detection of herpes simplex virus DNA in cutaneous lesions of erythema multiforme. J Invest Dermatol 93(1): 183-187.

20. Schofield JK, Tatnall FM, Brown J, McCloskey D, Navarrete C, et al (1994) Recurrent erythema multiforme: Tissue typing in a large series of patients. Br J Dermatol 131(4): 532-535.
21. Farthing P, Bagan JV, Scully C (2005) Mucosal disease series. Number IV. Erythema multiforme. Oral Dis 11(5): 261-267.

22. Iwai K, Miyawaki T, Takizawa T, Konno A, Otha K, et al. (1994) Differential expression of bcl-2 and susceptibility to anti-Fas-mediated cell death in peripheral blood lymphocytes, monocytes, and neutrophils. Blood 84(4): 1201-1208.

23. Sayama K, Yonehara S, Watanabe Y, Miki Y (1994) Expression of Fas antigen on keratinocytes in vivo and induction of apoptosis in cultured keratinocytes. J Invest Dermatol 103(3): 330-334.

Your next submission with Juniper Publishers will reach you the below assets

- Quality Editorial service

- Swift Peer Review

- Reprints availability

- E-prints Service

- Manuscript Podcast for convenient understanding

- Global attainment for your research

- Manuscript accessibility in different formats ( Pdf, E-pub, Full Text, Audio)

- Unceasing customer service

Track the below URL for one-step submission https://juniperpublishers.com/online-submission.php 